\title{
Long-term cardiac-specific mortality among 44,292 acute myeloid leukemia patients treated with chemotherapy: a population-based analysis
}

\author{
Guangli Li 1* , Zhijuan Zhou ${ }^{1,2^{*}}$, Wencong Yang ${ }^{3^{*}}$, Hao Yang ${ }^{1}$, Xiuwu Fan ${ }^{1}$, Yuelan Yin ${ }^{1,2}$, Liyun Luo ${ }^{1,2}$, \\ Jinyou Zhang ${ }^{1}$, Niujian Wu ${ }^{1}$, Zibin Liang ${ }^{\natural}$, Jianting Ke ${ }^{5 \bowtie}$, Jian Chen ${ }^{1,2,6}$ \\ 1. Department of Cardiology, The Fifth Affiliated Hospital, Sun Yat-sen University, Zhuhai 519000, China. \\ 2. Center for Interventional Medicine, The Fifth Affiliated Hospital, Sun Yat-sen University, Zhuhai 519000, China. \\ 3. Department of Cardiology, The Seventh Affiliated Hospital, Sun Yat-sen University, Shenzhen 518017, Guangdong, China. \\ 4. Department of Thoracic Oncology, The Cancer Center of The Fifth Affiliated Hospital of Sun Yat-sen University, Zhuhai 519000, China. \\ 5. Department of Nephrology, The Fifth Affiliated Hospital, Sun Yat-sen University, Zhuhai 519000, China. \\ 6. Guangdong Provincial Key Laboratory of Biomedical Imaging, The Fifth Affiliated Hospital, Sun Yat-sen University, Zhuhai 519000, China. \\ ${ }^{*}$ Dr. Guangli Li, Zhijuan Zhou and Wencong Yang have equally contributed to this article.
}

$\triangle$ Corresponding authors: Zibin Liang, M.D. \& Ph.D., Department of Thoracic Oncology, The Cancer Center of The Fifth Affiliated Hospital of Sun Yat-sen University, Zhuhai 519000, China. (email: liangzb@mail.sysu.edu.cn); Or Jianting Ke, M.D. \& Ph.D., Department of Nephrology, The Fifth Affiliated Hospital, Sun Yat-sen University, Zhuhai 519000, China. (email: kejt@mail.sysu.edu.cn); Or Jian Chen, M.D. \& Ph.D., Department of Cardiology; Center for Interventional Medicine; Guangdong Provincial Key Laboratory of Biomedical Imaging, The Fifth Affiliated Hospital, Sun Yat-sen University, Zhuhai 519000, China. (email: chenjn@mail.sysu.edu.cn)

(c) The author(s). This is an open access article distributed under the terms of the Creative Commons Attribution License (https://creativecommons.org/licenses/by/4.0/). See http://ivyspring.com/terms for full terms and conditions.

Received: 2019.05.23; Accepted: 2019.08.21; Published: 2019.10.15

\begin{abstract}
Background: Acute myeloid leukemia $(\mathrm{AML})$ is a common hematological malignancy treated with regimens containing anthracycline, an agent with cardiotoxicity. However, the cardiac-specific mortality in AML patients receiving chemotherapy remains unknown.

Methods: In this population-based study, patients diagnosed with AML between 1973 and 2015 were identified in the Surveillance, Epidemiology, and End Results database. Cumulative mortality by cause of death was calculated. To quantify the excessive cardiac-specific death compared with the general population, standardized mortality ratios (SMRs) were calculated. Multivariate Cox regression analyses were performed to identify risk factors associated with cardiac-specific death and AML-specific death.

Results: A total of 64,679 AML patients were identified between 1973 and 2015; 68.48\% of patients $(44,292)$ received chemotherapy. Among all possible competing causes of death, AML was associated with the highest cumulative mortality. The AML patients who received chemotherapy showed excessive cardiac-specific mortality compared with the general population, with an SMR of 6.35 (95\% CI: 5.89-6.82). Age, year of diagnosis, sex, and marital status were independently associated with patient prognosis.

Conclusion: Cardiac-specific mortality in AML patients receiving chemotherapy is higher than that in the general population.
\end{abstract}

Key words: acute myeloid leukemia, chemotherapy, cardiac-specific death, epidemiology and cohort study.

\section{Introduction}

Acute myeloid leukemia (AML) is a malignancy originating from abnormally differentiated blasts of myeloid lineage [1]. It has been estimated that 61,780 new leukemia cases and 22,840 leukemia-related deaths will be observed in the U.S.A. in 2019 [2]. Previous reports showed that AML usually occurs in elderly adults, of whom the median age is 67 years, and approximately $30 \%$ of AML patients are over 75 years old [3]. Over the past decades, development in hematopoietic stem cell transplantation, supportive care and salvage chemotherapy has led to the prolongation of patient survival $[4,5]$. The first-line 
induction chemotherapy regimen for $A M L$, except for acute promyelocytic leukemia (APL), has been the daunorubicin and cytarabine (DA) regimen for more than three decades, and this anthracycline-containing regimen is believed to exert cardiac toxicity [6]. Previous studies on the adverse effect of anthracycline on heart physiology demonstrated that it may cause arrhythmia and congestive heart failure in patients with breast cancer and Hodgkin lymphoma [7-11]. Furthermore, a recent publication demonstrated that it may lead to left ventricular function impairment in children survivors of AML [12]. The treatment of APL has developed dramatically over the past three decades due to the elucidation of the PML/RAR fusion gene, from anthracycline monotherapy to arsenic-based therapy to a combinatorial therapy of arsenic and all-trans retinoic acid [13]. However, arsenic agents have been proven to impair cardiac electrophysiology and ultimately increase cardiacspecific mortality [14, 15]. Therefore, it is thought that chemotherapy is of high risk for AML patients, a population with many senior citizens [16]. Despite the fact that agents with heart toxicity are frequently used in AML, quantitative analyses of cardiac-specific mortality based on large sample sizes have not been reported. Thus, it is of great importance and urgency to clarify the cardiac-specific mortality in AML patients receiving chemotherapy.

Previous reports in this field mainly focused on the molecular interactions of heart toxicity, potential biomarkers, and preventive agents or were simply case reports [17-23]. It is also important to clarify the epidemiology of chemotherapy-related cardiacspecific death in AML patients to find some clues for basic research. The current study utilized data from the population-based Surveillance, Epidemiology, and End Results (SEER) database to report cardiac-specific mortality by age and to identify risk factors.

\section{Materials and methods}

\section{Case inclusion}

We accessed the SEER database, which is maintained and updated by the National Cancer Institute, to enroll AML cases. For all incident cases in the coverage areas, the SEER registries collect clinicopathological and demographic information, such as sex, race, age, socioeconomic status (SES), survival time, and vital status [24]. The 18 registries of the SEER database cover approximately $28 \%$ of the total population, with nine registries covering $9 \%$ of the total population [25].

Data collection was performed by National Cancer Institute SEER*Stat software (seer.cancer.gov/ seerstat) version 8.3.2. Cases of AML were histologically identified by the following ICD-O-3 codes: $9840 / 3,9860 / 3,9861 / 3$, NOS, 9865/3, 9866/3, 9867/3, 9869/3, 9870/3, 9871/3, 9872/3, 9873/3, 9874/3, 9891/3, 9895/3, 9896/3, 9897/3, 9898/3, $9910 / 3,9911 / 3,9920 / 3$ and 9931/3. We identified patients with a first malignancy diagnosis of AML between 1 January 1973 and 31 December 2015. We excluded patients who were diagnosed only by death certificate or autopsy report and patients whose age, race, SES status or survival time were unknown. The county-level SES was used as a surrogate of personal SES for each case and was defined as the percentage of residents living below the national poverty threshold in the 2000 U.S. Census [26-28]. Additionally, three levels of SES were defined by thresholds recommended by the National Cancer Institute: $<10 \%$ for low-poverty areas, $10 \%-19.99 \%$ for medium-poverty areas, and $>20 \%$ for high-poverty areas [29].

\section{Statistical analyses}

The baseline characteristics of the included cases are summarized in Table 1, with frequencies for categorical variables and means and corresponding standard deviations for continuous variables; their differences were tested by chi-square analyses and unpaired $t$ tests, respectively. Cumulative mortality was computed for each cause of death and grouped by age. As the mortality of cardiac-specific diseases increases significantly with age in the general population, we calculated the standardized mortality ratios (SMRs) with respect to the mortality data for each age category of the general population in the U.S.A. to address this problem [30]. We divided the observed number of cardiac-specific deaths by the expected number in the same age category, which was obtained by multiplying the cumulative person-time in AML patients in an age group with the age-specific cardiac-specific mortality rates for the general population in the same age group. All age-specific SMRs were computed for patients older than 50 years receiving chemotherapy. The 95\% confidential intervals and p-values were also calculated [31, 32]. Multivariate Cox regression analyses were performed to identify the risk factors for both cardiac-specific and AML-specific death. All other analyses were performed with Stata software, version 14.0 (StataCorp. 2015. Stata Statistical Software: Release 14. College Station, TX: StataCorp LP.). A two-sided p < 0.05 was considered statistically significant.

\section{Results}

\section{Baseline characteristics}

Of the 64,679 patients diagnosed with acute 
myeloid leukemia (AML) between 1973 and 2015, $68.48 \%(44,292)$ received chemotherapy, and $31.52 \%$ of patients $(20,387)$ did not receive chemotherapy (Table 1). Patients who received chemotherapy were generally younger at diagnosis, with an average age of diagnosis of 54 years, compared with those in the nonchemotherapy group (average age: 73 years old). Additionally, about $86 \%$ of patients in the nonchemotherapy group were older than 60 years compared with $47.48 \%$ in the chemotherapy group. The majority of patients were white, and the sex, socioeconomic status (SES) and marital status distributions were relatively homogeneous in both groups. In terms of the calendar period of diagnosis, patients diagnosed in the last decade were more likely to receive chemotherapy than those diagnosed prior (46.22\% vs. 39.92\%). Generally, patients who received chemotherapy had a higher probability of survival $(26.74 \%$ vs. $4.85 \%)$ and a lower probability of dying from AML $(62.39 \%$ vs. $78.36 \%)$ or heart disease $(1.71 \%$ vs. $4.19 \%)$.

Table 1. Basic characteristics of all AML cases by chemotherapy status between 1973 and 2015. Data are number of patients in each category, with percentage in parentheses.

\begin{tabular}{|c|c|c|c|c|}
\hline \multirow[t]{2}{*}{ Category } & \multirow[t]{2}{*}{ All patients(n, \%) } & \multicolumn{2}{|c|}{ Chemotherapy(n, \%) } & \multirow{2}{*}{$\begin{array}{l}\mathrm{P} \\
\text { value }\end{array}$} \\
\hline & & yes & no & \\
\hline & 64679 & $44292(68.48)$ & $20387(31.52)$ & \\
\hline \multicolumn{5}{|l|}{ Age } \\
\hline Mean \pm SD & $59.78 \pm 21.96$ & $53.56 \pm 21.59$ & $73.29 \pm 15.84$ & $<0.01$ \\
\hline $0-14$ & $3216(4.97)$ & $3004(6.78)$ & $212(1.04)$ & $<0.01$ \\
\hline $15-29$ & $4406(6.81)$ & $4037(9.11)$ & $369(1.81)$ & \\
\hline $30-44$ & $6727(10.40)$ & $6067(13.70)$ & $660(3.24)$ & \\
\hline $45-59$ & $11769(18.20)$ & $10156(22.93)$ & $1613(7.91)$ & \\
\hline $60-74$ & 19553 (30.23) & $14000(31.61)$ & $5553(27.24)$ & \\
\hline $75+$ & 19008 (29.39) & 7028 (15.87) & $11980(58.76)$ & \\
\hline \multicolumn{5}{|l|}{ Race } \\
\hline White & $53766(83.13)$ & $36319(82.00)$ & $17447(85.58)$ & $<0.01$ \\
\hline Black & $5342(8.26)$ & $3851(8.69)$ & $1491(7.31)$ & \\
\hline Other & $5335(8.25)$ & $4007(9.05)$ & $1328(6.51)$ & \\
\hline Unknown & $236(0.36)$ & $115(0.26)$ & $121(0.59)$ & \\
\hline \multicolumn{5}{|l|}{ Sex } \\
\hline Male & 35091 (54.25) & 24395 (55.08) & $10696(52.46)$ & $<0.01$ \\
\hline Female & $29588(45.75)$ & 19897 (44.92) & $9691(47.54)$ & \\
\hline \multicolumn{5}{|l|}{ SES } \\
\hline Low & $26922(41.62)$ & $18469(41.70)$ & 8453 (41.46) & $=0.25$ \\
\hline Medium & $33693(52.09)$ & $23087(52.12)$ & $10606(52.02)$ & \\
\hline High & $4064(6.28)$ & $2736(6.18)$ & $1328(6.51)$ & \\
\hline \multicolumn{5}{|l|}{ Marriage } \\
\hline Married & $34442(53.25)$ & $24724(55.82)$ & 9718 (47.67) & $<0.01$ \\
\hline Unmarried & $27659(42.76)$ & $18286(41.29)$ & $9373(45.98)$ & \\
\hline Unknown & $2578(3.99)$ & $1282(2.89)$ & $1296(6.36)$ & \\
\hline \multicolumn{5}{|l|}{ Year } \\
\hline 1973-1985 & 7991 (12.35) & $5138(11.60)$ & 2853 (13.99) & $<0.01$ \\
\hline 1986-1995 & $8138(12.58)$ & 5551 (12.53) & 2587 (12.69) & \\
\hline 1996-2005 & $19939(30.83)$ & $13131(29.65)$ & 6808 (33.39) & \\
\hline 2006-2015 & $28611(44.24)$ & $20472(46.22)$ & 8139 (39.92) & \\
\hline \multicolumn{5}{|c|}{ Cause of death } \\
\hline Alive & $12831(19.84)$ & $11842(26.74)$ & 989 (4.85) & $<0.01$ \\
\hline AML & 43609 (67.42) & 27633 (62.39) & $15976(78.36)$ & \\
\hline Cardiac & $1614(2.50)$ & $759(1.71)$ & 855 (4.19) & \\
\hline Other & $6625(10.24)$ & 4058 (9.16) & 2567 (12.59) & \\
\hline
\end{tabular}

\section{Cumulative mortality}

The cumulative mortalities of AML patients receiving chemotherapy are illustrated by causes of death in Figure 1. AML was the major cause of death with the highest mortality, followed by other cancers, other noncancer diseases and heart disease. In addition, the heart disease mortality marginally increased over the 15-year period after diagnosis. In all age groups, the highest mortality rates were found in patients whose deaths were attributed to AML (Figure 2). Intuitively, the cumulative mortality of cardiac-specific disease increased with age at diagnosis and follow-up time. In terms of cardiac-specific cumulative mortality for AML patients who received chemotherapy, male patients showed higher cumulative mortality than female patients (Figure 3A). In terms of age, higher age was intuitively associated with higher cumulative mortality, with dramatically higher mortality in patients aged 60-74 and 75+ years (Figure 3B). White patients and Black patients showed similar mortality compared with the lower mortality in patients of other races (Figure 3C). Patients from medium-poverty regions showed higher mortality than those from low-poverty regions (Figure 3D).

\section{Standardized mortality ratios}

The standardized cardiac-specific mortality of AML patients was calculated with regard to the general population (Table 2). For cardiac-specific death among AML patients receiving chemotherapy, the SMRs were dramatically high in AML patients for most age groups, with an overall cardiac-specific mortality of $6.35 \quad(95 \% \quad \mathrm{CI}$ : 5.89-6.82). After stratification by age, the SMR decreased with age, from 11.03 (95\% CI: 7.91-14.15) in patients aged 50-54 to 1.25 (95\% CI: $0.95-1.56)$ in patients aged $80-84$. Counterintuitively, patients who received chemotherapy showed a lower SMR of 2.26 (95\% CI: 2.10-2.42) compared with counterparts who did not receive chemotherapy (SMR of 5.53, 95\% CI: 5.27-5.79). In terms of all-cause mortality, the overall SMR was 95.80 (95\% CI: 94.74-96.86) in the AML population. Similar decreasing trends of SMRs with age were also observed, from 144.13 in patients aged 50-54 to 15.08 in patients aged 80-84. Patients who received chemotherapy showed a lower SMR of 96.62 (95\% CI: 95.57-97.67) compared with their nonchemotherapy counterparts with (SMR of 125.49, 95\% CI: 124.24-126.73). 
Cause of death

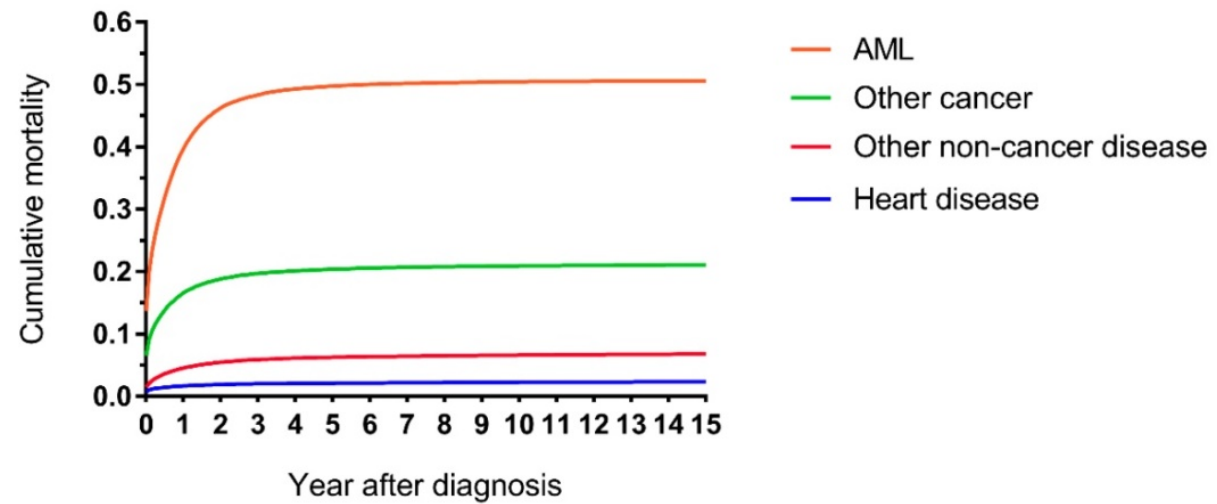

Figure 1. Cumulative mortality by causes of death in patients with AML receiving chemotherapy from 1973 to 2015 in SEER 18 registries.

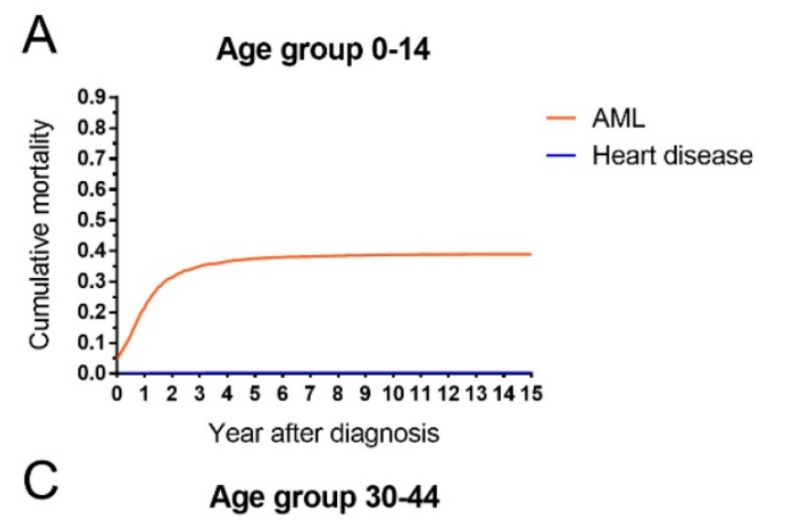

B Age group15-29
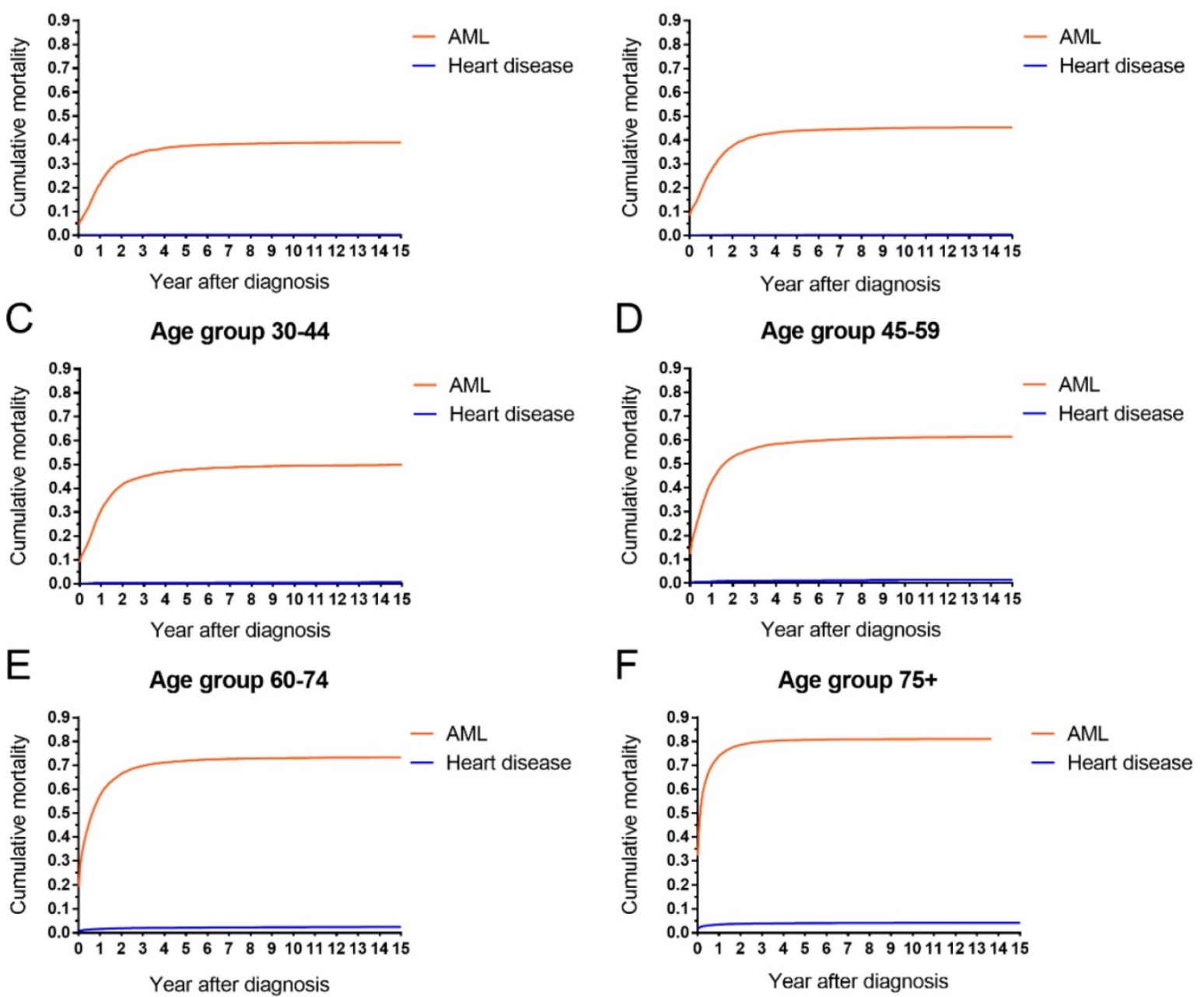

Figure 2. AML-specific and cardiac-specific mortality by age in patients with AML receiving chemotherapy from 1973 to 2015 in SEER 18 registries. 
A

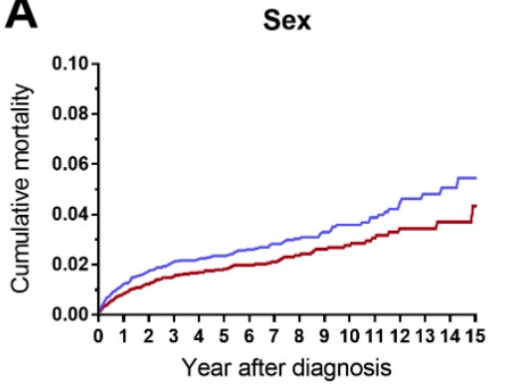

C

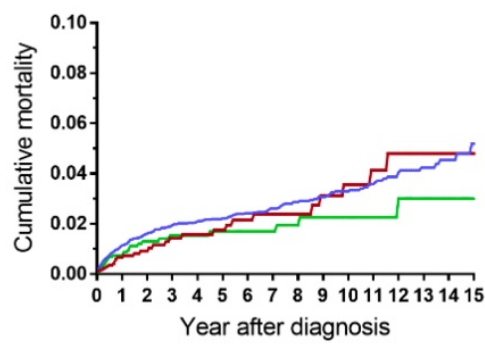

B

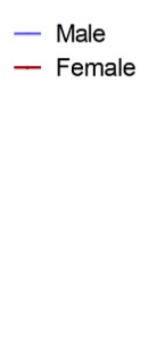

- Whites

- Blacks

Figure 3. Differences in cardiac-specific mortality by sex, age, race and socioeconomic status in patients with AML receiving chemotherapy from 1973 to 2015 in SEER 18 registries.

Table 2. Standardized mortality rates for AML patients receiving chemotherapy by age and for all AML patients by chemotherapy status, relative to the USA general population. Data are standardized mortality rates, with $95 \%$ confidence interval in parentheses.

\begin{tabular}{lllll}
\hline Groups & $\begin{array}{l}\text { SMR } \\
\text { cardiac-specific }\end{array}$ & P value & SMR overall & P value \\
\hline $\begin{array}{l}\text { Overall } \\
\text { Age }\end{array}$ & $6.35(5.89-6.82)$ & $<0.0001$ & $95.80(94.74-96.86)$ & $<0.0001$ \\
$50-54$ & $11.03(7.91-14.15)$ & $<0.0001$ & $144.13(138.33-149.92)$ & $<0.0001$ \\
$55-59$ & $9.98(7.74-12.23)$ & $<0.0001$ & $107.54(103.65-111.43)$ & $<0.0001$ \\
$60-64$ & $6.13(4.82-7.44)$ & $<0.0001$ & $77.76(75.24-80.28)$ & $<0.0001$ \\
$65-69$ & $5.11(4.16-6.05)$ & $<0.0001$ & $55.80(54.09-57.50)$ & $<0.0001$ \\
$70-74$ & $3.26(2.66-3.86)$ & $<0.0001$ & $37.61(36.47-38.75)$ & $<0.0001$ \\
$75-79$ & $1.86(1.48-2.24)$ & $<0.0001$ & $24.13(23.32-24.94)$ & $<0.0001$ \\
$80-84$ & $1.25(0.95-1.56)$ & $=0.077$ & $15.08(14.43-15.73)$ & $<0.0001$ \\
$\begin{array}{l}\text { Treatment } \\
\text { Chemotherapy }\end{array}$ & $2.26(2.10-2.42)$ & $<0.0001$ & $96.62(95.57-97.67)$ & $<0.0001$ \\
$\begin{array}{l}\text { No } \\
\text { chemotherapy }\end{array}$ & $5.53(5.27-5.79)$ & $<0.0001$ & $125.49(124.24-126.73)$ & $<0.0001$ \\
\hline
\end{tabular}

\section{Cause-specific hazard ratios}

The correlation between clinicopathological characteristics and either cardiac-specific or AML-specific causes of death for patients who received chemotherapy is demonstrated in Table 3 . Increased age was associated with higher risk for cardiac-specific death, with hazard ratios (HRs) of 1.34 (95\% CI: 0.69-2.60), 3.33 (95\% CI: 1.85-6.00), 8.91 (95\% CI: 5.13-15.48), 26.45 (95\% CI: 15.29-45.75), and 62.63 (95\% CI: 35.83-109.48) for patients aged 15-29, 30-44, 45-59, 60-74, and 75+, respectively, compared with patients aged 0-14. However, the impact of age on AML-specific mortality was weaker than on cardiac-specific mortality, with hazard ratios of 1.22 (95\% CI: 1.13-1.32), 1.53 (95\% CI: 1.42-1.64), 2.35 (95\%
CI: $2.19-2.52), 3.83$ (95\% CI: 3.58-4.10), and 6.43 (95\% CI: 6.00-6.90) for patients aged 15-29, 30-44, 45-59, 60-74, and 75+, respectively, compared with patients aged 0-14. In terms of the year of diagnosis, intuitively, patients diagnosed in later decades are associated with lower hazard ratios for both cardiac-specific and AML-specific mortality. For cardiac-specific mortality, hazard ratios were 0.71 (95\% CI: 0.55-0.92), 0.46 (95\% CI: 0.36-0.58), and 0.42 (95\% CI: $0.33-0.53$ ) for patients diagnosed in 1986-1995, 1996-2005, and 2006-2015, respectively, with patients diagnosed in 1973-1985 as a reference. For AML-specific mortality, hazard ratios were 0.75 (95\% CI: 0.72-0.79), 0.58 (95\% CI: 0.56-0.60), and 0.42 (95\% CI: $0.40-0.44)$ for patients diagnosed in 1986-1995, 1996-2005, and 2006-2015, respectively, with patients diagnosed in 1973-1985 as a reference. No difference in risk of cardiac-specific mortality was observed among races. For AML-specific mortality, Black patients showed a marginally higher hazard ratio of 1.09 (95\% CI: 1.04-1.14) compared with the hazard ratio of 0.55 (95\% CI: $0.37-0.80)$ for patients of unknown race. In terms of sex, females showed lower hazard ratios in both cardiac-specific and AML-specific mortality (0.67 (95\% CI: 0.57-0.79) and 0.89 (95\% CI: 0.87-0.91), respectively), compared with males. For SES, a higher risk of AML-specific death was found in the medium- and high-poverty groups, with HRs of 1.04 (95\% CI: 1.01-1.07) and 1.17 (95\% CI: 1.11-1.24), respectively. In terms of marital status, unmarried patients were associated with a higher risk of cardiac-specific death, with an HR of 1.13 (95\% CI: 1.10-1.16). 
Table 3. Multivariate Cox regression analyses for AML patients with chemotherapy by causes of death. Data are hazard ratios, with $95 \%$ confidence interval in parentheses.

\begin{tabular}{|c|c|c|c|c|c|}
\hline \multirow[t]{2}{*}{ Strata } & \multirow[t]{2}{*}{$\mathrm{N}$} & \multicolumn{4}{|c|}{ Cause-specific hazards ratios } \\
\hline & & Cardiac & P-value & AML & P-value \\
\hline \multicolumn{6}{|l|}{ Age } \\
\hline $0-14$ & 3004 & Reference & & Reference & \\
\hline $15-29$ & 4037 & $1.34(0.69-2.60)$ & 0.388 & $1.22(1.13-1.32)$ & $<0.01$ \\
\hline $30-44$ & 6067 & $3.33(1.85-6.00)$ & $<0.01$ & $1.53(1.42-1.64)$ & $<0.01$ \\
\hline $45-59$ & 10156 & $8.91(5.13-15.48)$ & $<0.01$ & $2.35(2.19-2.52)$ & $<0.01$ \\
\hline $60-74$ & 14000 & $26.45(15.29-45.75)$ & $<0.01$ & $3.83(3.58-4.10)$ & $<0.01$ \\
\hline $75+$ & 7028 & $62.63(35.83-109.48)$ & $<0.01$ & $6.43(6.00-6.90)$ & $<0.01$ \\
\hline \multicolumn{6}{|l|}{ Year } \\
\hline 1973-1985 & 5138 & Reference & & Reference & \\
\hline 1986-1995 & 5551 & $0.71(0.55-0.92)$ & $<0.01$ & $0.75(0.72-0.79)$ & $<0.01$ \\
\hline 1996-2005 & 13131 & $0.46(0.36-0.58)$ & $<0.01$ & $0.58(0.56-0.60)$ & $<0.01$ \\
\hline 2006-2015 & 20472 & $0.42(0.33-0.53)$ & $<0.01$ & $0.42(0.40-0.44)$ & $<0.01$ \\
\hline \multicolumn{6}{|l|}{ Race } \\
\hline White & 36319 & Reference & & Reference & \\
\hline Black & 3851 & $1.01(0.75-1.36)$ & 0.934 & 1.09 (1.04-1.14) & $<0.01$ \\
\hline Other & 4007 & $1.01(0.77-1.33)$ & 0.939 & $1.03(0.98-1.08)$ & 0.223 \\
\hline Unknown* & 115 & - & - & $0.55(0.37-0.80)$ & $<0.01$ \\
\hline \multicolumn{6}{|l|}{ Sex } \\
\hline Male & 24395 & Reference & & Reference & \\
\hline Female & 19897 & $0.67(0.57-0.79)$ & $<0.01$ & $0.89(0.87-0.91)$ & $<0.01$ \\
\hline \multicolumn{6}{|l|}{ SES } \\
\hline Low & 18469 & Reference & & Reference & \\
\hline Medium & 23087 & $1.11(0.95-1.31)$ & 0.202 & $1.04(1.01-1.07)$ & $<0.01$ \\
\hline High & 2736 & $1.42(1.01-2.00)$ & 0.044 & $1.17(1.11-1.24)$ & $<0.01$ \\
\hline \multicolumn{6}{|l|}{ Marriage } \\
\hline Married & 24724 & Reference & & Reference & \\
\hline Unmarried & 18286 & $1.56(1.32-1.85)$ & $<0.01$ & $1.13(1.10-1.16)$ & $<0.01$ \\
\hline Unknown & 1282 & $1.42(0.90-2.23)$ & 0.13 & $0.97(0.89-1.05)$ & 0.443 \\
\hline
\end{tabular}

\section{Discussion}

The current study is the first to demonstrate the mortality of AML patients by cause of death and extrapolate the cardiac-specific mortality. We found that cardiac-specific mortality accounts for approximately $2.50 \%$ of AML patient deaths, with a standardized mortality ratio of 6.35 , implying a higher risk of death from heart disease compared with the general population.

Here, higher cardiac-specific mortality in AML patients who received chemotherapy was confirmed with an SMR of 6.35 compared with the general population. AML patients show increased cardiac-specific mortality as a result of both tumor cell infiltration in heart tissue and the administration of cardiotoxic chemotherapy, especially anthracyclinecontaining induction therapy [33-35]. The cardiotoxicity of anthracycline increases in a cumulative dose-related manner, and therefore, a maximally tolerated dose was set as a cumulative dose of $550 \mathrm{mg} / \mathrm{m} 2$ to achieve a balance between tumor control and cardiac function impairment [36]. In a study of induction chemotherapy, chemotherapy and cytokines released from the lysis of tumor cells may have instigated congestive heart failure in 18 out of 217 patients $(8.29 \%)$; 5 of these patients recovered, whereas the remaining 13 patients did not (median survival of 71 days) [37]. Basic research studies have shown that anthracycline can also cause myocyte necrosis $[38,39]$. These diverse detrimental effects are associated with polymorphisms of anthracycline metabolism enzymes, which makes tailored safety dosing possible $[12,21,40,41]$. Although the mechanism of cardiotoxicity in AML has not been clarified, studies in breast cancer patients whose chemotherapy regimens contain anthracycline demonstrated that the impairment of heart cells is mediated by stress protective signaling and reactive oxygen [42, 43]. Notably, cardiac damage does not necessarily lead to cardiac-specific death, and thus, the mortality observed here may underestimate the cardiac damage. Therefore, future studies using ultrasound, MRI or PET as surveillance methods are of importance to provide a more precise landscape of heart damage. To study the excessive cardiac mortality that might be induced by chemotherapy, we calculated the SMRs, taking into account the increasing mortality from heart diseases with age in the general population. In the current study, we found a significantly higher SMR in patients who received chemotherapy and in each age category. Counterintuitively, patients who received chemotherapy showed a lower SMR compared with patients without chemotherapy. This result must be interpreted with caution, as there are differences in baseline characteristics, with generally higher ages and higher unmarried rates, in patients without chemotherapy. It has been reported that patients who receive chemotherapy are generally younger and have fewer comorbidities and better health [44-46].

In multivariate Cox regression analyses, the risks of death from heart diseases and AML increased with age, which seemingly contradicts the decreasing SMRs. This can be partially explained by the fact that the SMR was calculated by dividing the observed number of deaths in the AML cohort by the expected number of deaths in the general population who also show increasing mortality with increasing age. In terms of sex, females showed a reduced risk of cardiac-specific death compared with males, implying intrinsic biological differences in cardiac-specific disease mortality [16, 47]. In terms of marital status, unmarried patients and patients with unknown marital status showed a higher risk of death compared with married patients, in line with a previous publication [48]. This survival disparity may be associated with the impact of better social support on treatment adherence; living in a close and cohesive family increases the probability of adherence 1.7-fold, whereas living in an unstable family increases the risk of nonadherence 1.5-fold [49]. In terms of SES, 
residing in high-poverty regions was marginally associated with a higher risk of cardiac-specific death, which implies poor healthcare accessibility in poor regions [50, 51]. In AML-specific mortality, elderly patients and patients diagnosed in early decades had a higher risk of death. The higher risk of death in elderly patients may be attributable to treatmentrelated mortality and resistance to chemotherapy [52, 53]. Elder patients are less able to tolerate intensive chemotherapy because of poor performance status, more comorbidities and poor immunity towards posttreatment infection. Additionally, older individuals show a high incidence of unfavorable genetic mutations and treatment-resistant phenotypes [54-56]. Importantly, Black patients have a higher risk of death due to treatment differences and unmeasured genetic differences associated with excessive mortality during induction $[57,58]$. A lower risk of AML-specific death in females may imply a protective role of estrogen $[59,60]$. Inferior survival in patients from medium-poverty and high-poverty regions implies the impact of SES on survival via the limitation of access to healthcare resources, such as adequate treatment and surveillance [26, 61, 62].

The current study has strengths as well as limitations. First, the SEER database does not provide detailed information about the chemotherapy regimen, chemotherapy duration or application of hematopoietic stem cell transplantation, which have also been proven to be harmful to heart function [63, 64]. Moreover, the SEER database does not provide detailed information, such as comorbidities, types of heart disease, and menopause status of females, which would be helpful for further analysis. Second, due to the long-term follow-up of these patients, the hazard ratios here imply the average risk of a specific group. Third, the results in the current study may be biased by confounding factors owing to its retrospective nature. As all cases came from the 18 registries of the SEER database, the epidemiological trend here can only reflect the pattern in the selected areas, and great heterogeneity exists in different patient populations in terms of AML biology (different mutation patterns), healthcare services, and chemotherapy dosages based on physicians' preference. Therefore, caution should be used when interpreting the current results to predict the epidemiology of AML in other regions. Fourth, the calculation of SMRs did not adjust for other confounders, as we did in the multivariate Cox regressions, which may confound these results. Fifth, the standard mortality data of the general population from CDC WONDER are provided in a combined manner, and the exclusion of persons diagnosed with AML was not possible. However, due to the low incidence of AML (nearly 4 per 100,000) in the general population, our results may not be substantially biased. Last, the representativeness of the SEER database for the general population in the U.S.A. is moderately questioned, as the population in SEER registries are shown to have a lower SES and more diverse composition of ethnic minorities [65].

In summary, the current study distinguishes itself by the large sample size and long follow-up time, which make up for the limitations of not having a randomized clinical trial on this topic. Studying the long-term cardiac-specific mortality in AML patients may help to better tailor clinical surveillance protocols and ultimately improve patient wellbeing.

\section{Acknowledgement}

This work was supported by Science and Technology Planning Project of Guangdong Province (2014A020212088) and Science and Technology Planning Project of Zhuhai City (Major Program, 2015B1031).

\section{Sources of Funding}

This work was supported by Science and Technology Planning Project of Guangdong Province (2014A020212088) and Science and Technology Planning Project of Zhuhai City (Major Program, 2015B1031).

\section{Availability of materials and data}

All data in the current study can be accessed from the Surveillance, Epidemiology, and End Results database.

\section{Competing Interests}

The authors have declared that no competing interest exists.

\section{References}

1. Short NJ, Rytting ME, Cortes JE. Acute myeloid leukaemia. Lancet. 2018; 392: 593-606.

2. Siegel RL, Miller KD, Jemal A. Cancer statistics, 2019. CA Cancer J Clin. 2019; 69: 7-34.

3. Podoltsev NA, Stahl M, Zeidan AM, Gore SD. Selecting initial treatment of acute myeloid leukaemia in older adults. Blood Rev. 2017; 31: 43-62.

4. Carraway HE. Improving Overall Survival in Older Adults With Acute Myeloid Leukemia: Subpopulations Matter. J Clin Oncol. 2018: Jco2018793539.

5. Reedijk AMJ, Klein K, Coebergh JWW, Kremer LC, Dinmohamed AG, de Haas $\mathrm{V}$, et al. Improved survival for children and young adolescents with acute myeloid leukemia: a Dutch study on incidence, survival and mortality. Leukemia. 2018; 33: 1349-59.

6. Burnett A, Wetzler M, Lowenberg B. Therapeutic advances in acute myeloid leukemia. J Clin Oncol. 2011; 29: 487-94.

7. Pinder MC, Duan Z, Goodwin JS, Hortobagyi GN, Giordano SH. Congestive heart failure in older women treated with adjuvant anthracycline chemotherapy for breast cancer. J Clin Oncol. 2007; 25: 3808-15.

8. Kremer LC, van Dalen EC, Offringa M, Ottenkamp J, Voute PA. Anthracycline-induced clinical heart failure in a cohort of 607 children: long-term follow-up study. J Clin Oncol. 2001; 19: 191-6.

9. McLean BA, Hansen R, Paterson DI, White JA, Oudit GY. Breast Cancer Patients Receiving Anthracycline Chemotherapy and Trastuzumab Have Biventricular Dysfunction and Reduced Heart Mass. J Am Coll Cardiol. 2018; 72: 1872-3. 
10. Weberpals J, Jansen L, Muller OJ, Brenner H. Long-term heart-specific mortality among 347476 breast cancer patients treated with radiotherapy or chemotherapy: a registry-based cohort study. Eur Heart J. 2018; 39: 3896-903.

11. van Nimwegen FA, Ntentas G, Darby SC, Schaapveld M, Hauptmann M, Lugtenburg PJ, et al. Risk of heart failure in survivors of Hodgkin lymphoma: effects of cardiac exposure to radiation and anthracyclines. Blood. 2017; 129: 2257-65

12. Jarfelt $\mathrm{M}$, Andersen $\mathrm{NH}$, Glosli $\mathrm{H}$, Jahnukainen $\mathrm{K}$, Jonmundsson GK, Malmros $\mathrm{J}$, et al. Cardiac function in survivors of childhood acute myeloid leukemia treated with chemotherapy only: a NOPHO-AML study. Eur J Haematol. 2016; 97: 55-62.

13. DeAngelo DJ. Tailored Approaches to Induction Therapy for Acute Promyelocytic Leukemia. J Clin Oncol. 2017; 35: 583-6.

14. Hai JJ, Gill H, Tse HF, Kumana CR, Kwong YL, Siu CW. Torsade de Pointes during oral arsenic trioxide therapy for acute promyelocytic leukemia in a patient with heart failure. Ann Hematol. 2015; 94: 501-3.

15. Takeshita A, Uehara A, Shinjo K, Naito K, Sahara N, Yamazaki K, et al. Impairment of heart rate variability control during arsenic trioxide treatment for acute promyelocytic leukemia. Leukemia. 2004; 18: 647-8.

16. Magnussen C, Niiranen TJ, Ojeda FM, Gianfagna F, Blankenberg S, Njolstad I, et al. Sex Differences and Similarities in Atrial Fibrillation Epidemiology, Risk Factors, and Mortality in Community Cohorts: Results From the BiomarCaRE Consortium (Biomarker for Cardiovascular Risk Assessment in Europe). Circulation. 2017; 136: 1588-97.

17. Vachhani P, Shin S, Baron J, Thompson JE, Wetzler M, Griffiths EA, et al. Dexrazoxane for cardioprotection in older adults with acute myeloid leukemia. Leuk Res Rep. 2017; 7: 36-9.

18. Horacek JM, Tichy M, Jebavy L, Pudil R, Ulrychova M, Maly J. Use of multiple biomarkers for evaluation of anthracycline-induced cardiotoxicity in patients with acute myeloid leukemia. Exp Oncol. 2008; 30: 157-9.

19. Anderlini P, Benjamin RS, Wong FC, Kantarjian HM, Andreeff M, Kornblau $\mathrm{SM}$, et al. Idarubicin cardiotoxicity: a retrospective study in acute myeloid leukemia and myelodysplasia. J Clin Oncol. 1995; 13: 2827-34.

20. Pena M, Serra J, Ribera JM. Cardiac arrest in a patient diagnosed with acute myeloid leukemia after the first dose of idarubicin. Med Clin (Barc). 2018; 151. 506-7.

21. Militaru A, Avram A, Cimpean AM, Iurciuc M, Matusz P, Lighezan D, et al. The Assessment of Left Ventricle Function and Subclinical Atherosclerosis in Patients with Acute Myeloid Leukemia. In vivo. 2018; 32: 1599-607.

22. Armenian SH, Ehrhardt MJ. Optimizing Cardiovascular Care in Children With Acute Myeloid Leukemia to Improve Cancer-Related Outcomes. J Clin Oncol. 2019; 37: 1-6.

23. Leptidis J, Aloizos S, Chlorokostas P, Gourgiotis S. Fatal cardiac tamponade as the first manifestation of acute myeloid leukemia. Am J Emerg Med. 2014; 32: 1294.e1-2.

24. Warren JL, Klabunde CN, Schrag D, Bach PB, Riley GF. Overview of the SEER-Medicare data: content, research applications, and generalizability to the United States elderly population. Med Care. 2002; 40: IV-3-18.

25. Park HS, Lloyd S, Decker RH, Wilson LD, Yu JB. Overview of the Surveillance, Epidemiology, and End Results database: evolution, data variables, and quality assurance. Curr Probl Cancer. 2012; 36: 183-90.

26. Wang S, Tang J, Sun T, Zheng X, Li J, Sun H, et al. Survival changes in patients with small cell lung cancer and disparities between different sexes, socioeconomic statuses and ages. Sci Rep. 2017; 7: 1339

27. Krieger N, Chen JT, Waterman PD, Soobader MJ, Subramanian SV, Carson R. Geocoding and monitoring of US socioeconomic inequalities in mortality and cancer incidence: does the choice of area-based measure and geographic level matter?: the Public Health Disparities Geocoding Project. Am J Epidemiol. 2002; 156 : 471-82

28. Krieger N, Chen JT, Waterman PD, Rehkopf DH, Subramanian SV. Race/ethnicity, gender, and monitoring socioeconomic gradients in health: a comparison of area-based socioeconomic measures--the public health disparities geocoding project. Am J Public Health. 2003; 93: 1655-71.

29. Ward E, DeSantis C, Robbins A, Kohler B, Jemal A. Childhood and adolescent cancer statistics, 2014. CA Cancer J Clin. 2014; 64: 83-103.

30. Centers for Disease Control and Prevention. CDC Wonder. http://wonder.cdc.gov/ (18 October 2018).

31. Vandenbroucke JP. A shortcut method for calculating the 95 percent confidence interval of the standardized mortality ratio. American Journal of Epidemiology. 1982; 115: 303-4.

32. Altman DG, Bland JM. How to obtain the P value from a confidence interval. BMJ. 2011; 343: d2304.

33. Rigamonti F, Beris P, Sanchez-Pareja A, Meyer P, Ashrafpoor G, Zaza S, et al. Atypical presentation of acute myeloid leukemia: cardiac myeloid sarcoma. Int J Hematol. 2009; 89: 693-8.

34. Jost E, Lorenzen J, Haage P, Bos G, Beelen D, Galm O, et al. Heart and muscle involvement by extra-medullary myeloid leukemia: a case report and review of the literature. Leuk Lymphoma. 2005; 46: 1819-24.

35. Makaryus AN, Tung F, Liu W, Mangion J, Kort S. Extensive neoplastic cardiac infiltration in a patient with acute myelogenous leukemia: role of echocardiography. Echocardiography. 2003; 20: 539-44

36. Lefrak EA, Pitha J, Rosenheim S, Gottlieb JA. A clinicopathologic analysis of adriamycin cardiotoxicity. Cancer. 1973; 32: 302-14.
37. Raval P, Kota V, Zakharia Y, Carter G, Christopherson $\mathrm{K}$, Awan FT, et al Congestive heart failure (CHF) in acute myeloid leukemia patients during induction. Journal of Clinical Oncology. 2013; 31: 7105-

38. Billingham ME, Mason JW, Bristow MR, Daniels JR. Anthracycline cardiomyopathy monitored by morphologic changes. Cancer Treat Rep. 1978; 62: 865-72.

39. Mackay B, Ewer MS, Carrasco CH, Benjamin RS. Assessment of anthracycline cardiomyopathy by endomyocardial biopsy. Ultrastruct Pathol. 1994; 18: 203-11.

40. Megias-Vericat JE, Martinez-Cuadron D, Herrero MJ, Alino SF, Poveda JL, Sanz MA, et al. Pharmacogenetics of Metabolic Genes of Anthracyclines in Acute Myeloid Leukemia. Curr Drug Metab. 2018; 19: 55-74.

41. Megias-Vericat JE, Montesinos P, Herrero MJ, Moscardo F, Boso V, Rojas L, et al. Impact of $\mathrm{ABC}$ single nucleotide polymorphisms upon the efficacy and toxicity of induction chemotherapy in acute myeloid leukemia. Leuk Lymphoma. 2017; 58: 1197-206.

42. Shenoy C, Klem I, Crowley AL, Patel MR, Winchester MA, Owusu C, et al. Cardiovascular complications of breast cancer therapy in older adults. Oncologist. 2011; 16: 1138-43.

43. Meattini I, Guenzi M, Fozza A, Vidali C, Rovea P, Meacci F, et al. Overview on cardiac, pulmonary and cutaneous toxicity in patients treated with adjuvant radiotherapy for breast cancer. Breast Cancer. 2017; 24: 52-62.

44. Sacks NC, Cyr PL, Louie AC, Liu Y, Chiarella MT, Sharma A, et al. Burden of Acute Myeloid Leukemia Among Older, Newly Diagnosed Patients: Retrospective Analysis of Data From the 2010-2012 Medicare Limited Data Set. Clin Ther. 2018; 40: 692-703.e2.

45. Master S, Mansour R, Devarakonda SS, Shi Z, Mills G, Shi R. Predictors of Survival in Acute Myeloid Leukemia by Treatment Modality. Anticancer Res. 2016; 36: 1719-27.

46. Forsythe A, Kwon CS, Bell T, Smith TA, Arondekar B. Health-related quality of life in acute myeloid leukemia patients not eligible for intensive chemotherapy: results of a systematic literature review. Clinicoecon Outcomes Res. 2019; 11: 87-98.

47. Bots SH, Peters SAE, Woodward M. Sex differences in coronary heart disease and stroke mortality: a global assessment of the effect of ageing between 1980 and 2010. BMJ Glob Health. 2017; 2: e000298-e.

48. Aizer AA, Chen M-H, McCarthy EP, Mendu ML, Koo S, Wilhite TJ, et al. Marital Status and Survival in Patients With Cancer. Journal of Clinical Oncology. 2013; 31: 3869-76.

49. DiMatteo MR. Social support and patient adherence to medical treatment: a meta-analysis. Health Psychol. 2004; 23: 207-18.

50. Cainzos-Achirica M, Capdevila C, Vela E, Cleries M, Bilal U, Garcia-Altes A, et al. Individual income, mortality and healthcare resource use in patients with chronic heart failure living in a universal healthcare system: A population-based study in Catalonia, Spain. Int J Cardiol. 2019; 277: 250-7.

51. Dokainish H, Teo K, Zhu J, Roy A, AlHabib KF, ElSayed A, et al. Global mortality variations in patients with heart failure: results from the International Congestive Heart Failure (INTER-CHF) prospective cohort study. The Lancet Global Health. 2017; 5: e665-e72.

52. Estey EH. Treatment of acute myeloid leukemia. Haematologica. 2009; 94: $10-6$.

53. Hiddemann W, Kern W, Schoch C, Fonatsch C, Heinecke A, Wörmann B, et al. Management of Acute Myeloid Leukemia in Elderly Patients. Journal of Clinical Oncology. 1999; 17: 3569-76.

54. Klepin HD, Balducci L. Acute myelogenous leukemia in older adults. Oncologist. 2009; 14: 222-32.

55. Leith CP, Kopecky KJ, Godwin J, McConnell T, Slovak ML, Chen IM, et al. Acute myeloid leukemia in the elderly: assessment of multidrug resistance (MDR1) and cytogenetics distinguishes biologic subgroups with remarkably distinct responses to standard chemotherapy. A Southwest Oncology Group study. Blood. 1997; 89: 3323-9.

56. Fialkow PJ, Singer JW, Raskind WH, Adamson JW, Jacobson RJ, Bernstein ID, et al. Clonal development, stem-cell differentiation, and clinical remissions in acute nonlymphocytic leukemia. N Engl J Med. 1987; 317: 468-73.

57. Ma Y, Mitchell BS, Rhoads K. Are Racial and Ethnic Disparities In Acute Myeloid Leukemia Due To Differences In Treatment? Blood. 2013; 122: 726-

58. Winestone LE, Getz KD, Miller TP, Wilkes JJ, Sack L, Li Y, et al. Racial Disparities in Pediatric Acute Myeloid Leukemia during Induction. Blood. 2015; 126: 530

59. Rota S-G, Roma A, Dude I, Ma C, Stevens R, MacEachern J, et al. Estrogen Receptor $\beta$ Is a Novel Target in Acute Myeloid Leukemia. Molecular Cancer Therapeutics. 2017; 16: 2618-26.

60. Sánchez-Aguilera A, Méndez-Ferrer S. Regulation of hematopoietic progenitors by estrogens as a basis for new antileukemic strategies. Mol Cell Oncol. 2015; 3: e1009728-e.

61. Lu G, Li J, Wang S, Pu J, Sun H, Wei Z, et al. The fluctuating incidence, improved survival of patients with breast cancer, and disparities by age, race, and socioeconomic status by decade, 1981-2010. Cancer Manag Res. 2018; 10: 4899-914.

62. Borate UM, Mineishi S, Costa LJ. Nonbiological factors affecting survival in younger patients with acute myeloid leukemia. Cancer. 2015; 121:3877-84.

63. Vandekerckhove K, De Waele K, Minne A, Coomans I, De Groote K, Panzer J, et al. Evaluation of cardiopulmonary exercise testing, heart function, and quality of life in children after allogenic hematopoietic stem cell transplantation. Pediatr Blood Cancer. 2019; 66: e27499. 
64. Rotz SJ, Ryan TD, Jodele S, Jefferies JL, Lane A, Pate A, et al. The injured heart: early cardiac effects of hematopoietic stem cell transplantation in children and young adults. Bone Marrow Transplant. 2017; 52: 1171-9.

65. Kuo TM, Mobley LR. How generalizable are the SEER registries to the cancer populations of the USA? Cancer Causes Control. 2016; 27: 1117-26. 